\title{
Mechanical energy converter study for lotic water sensor applications
}

\author{
T. J. Schröder, Ch. Schöberlein, Ch. Schmidt, T. Keutel, O. Kanoun \\ Chair for Measurement and Sensor Technology, Technische Universität Chemnitz \\ Email: thomas.keutel@etit.tu-chemnitz.de
}

\begin{abstract}
Wireless sensor systems can help to improve or to expand instrumentation for environmental monitoring systems. For a autonomous power supply of such sensors, a mechanical converter solution will be proposed to convert energy using natural turbulences ever-present in lotic water. This contribution focusses $(a)$ on the mechanical-to-electrical energy conversion using a piezoelectric bimorph and $(b)$ on a suitable DC-DC converter supplying the wireless node built around the commercially available LTC3331 by Linear Technologies.

Index Terms-lotic water monitoring, wireless sensor node, mechanical energy converter, energy harvesting
\end{abstract}

\section{INTRODUCTION}

For a better understanding or controlling of environmental events like climate phenomena, pollution in general or human effected processes, wireless sensor systems provide an attractive opportunity to improve or to expand existing instrumentation in an economical way [12], [13]. An extended data base supports a better analysis and modelling of the mentioned phenomena for a more reliable prediction allowing a better preparation to prevent civil disasters in the future. Furthermore, additional information provides the opportunity to react quickly and more precise for example in cases of the contamination of lotic water, to prevent the spread of pollution. Therefore distributed sensor nodes are required for a flexible application, reliable and easily operable.

Within this contribution, general development steps will be presented for the design of a wireless sensor node (WSN) to monitor as an example lotic water quantities autonomously. The application field of small and medium streams where selected, because the limited availability of instruments in that field. One major challenge is the realization of a mechanical energy converter for powering the system using the kinetic energy of the lotic water.

The study contains an evaluation of possible energy sources, a study of methods to couple the mechanical energy of the lotic water including FEM analysis, empirical investigations using a flow passage and real environments. Furthermore, the system design of a WSN including an energy management, low power sensors and a unidirectional wireless communication will be discussed.

\section{CONCEPT OF THE SENSOR SYSTEM}

A general structure of a wireless sensor node is shown in Fig. 1. It illustrates the cooperation of energy converters and storage devices to supply a load, typical including sensors and a wireless communication, more briefly described in [15], [16]. One or multiple harvesters provide the energy

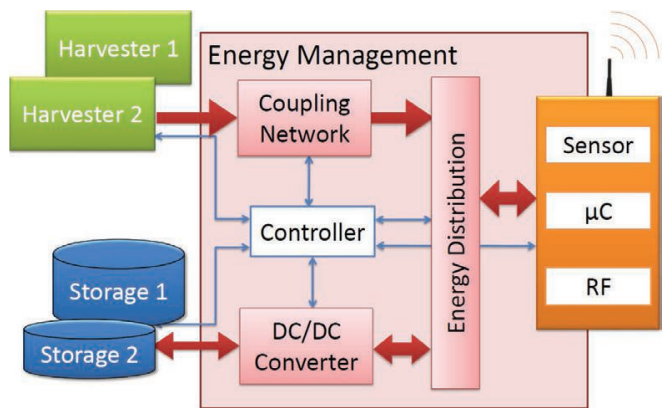

Fig. 1. Concept of a wireless sensor node

for the system. The energy flow and the energy distribution are controlled by the energy management. If the amount of energy harvested is not enough to power the system, an energy storage has to be employed. This working principle is mainly implemented in WSN solutions and is needed to consider for the development of such systems. This working principle is mainly implemented in WSN solutions and is to be considered for the development of such systems.

In lotic waters, energy can be present in the form of

- kinetic energy of the flowing water,

- temperature gradient between water and the ambiance,

- solar energy.

Considering the application scenario (lotic water environments) the flow of the water is the only source which is always available for energy harvesting. Thus, this method is preferred as a stable energy source.

For the conversion of the kinetic energy of turbulences into electrical energy a piezoelectric bimorph (MIDE Volture V21 BL, see fig. 2) was selected, which is available in different dimensions. Normally, these converters are used for

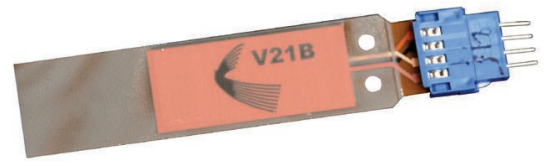

Fig. 2. Photograph of the piezoelectric bimorph used in this work energy harvesting from ambient vibrations, as described for 
example in [10], [11], [14]. Because of the specific electrical characteristics of the converter [17], a specific impedance matching is required to provide the generated energy. The implementation of the mechanical converter energy harvester and the development of the electronics will be discussed in detail in III-A (conversion of mechanical to electrical energy). The sensor, microcontroller and RF part will be discussed in detail in III-B. The sensor system will be completed with the implementation of a temperature sensor and an unidirectional wireless data transmission, delivering sensor data to a central receiver station.

The electrical power generated by the piezoelectric bimorph has to be high enough to overcome the leakage currents of the energy management section and should facilitate a wireless data transmission cycle of each 15 minutes.

\section{DISCUSSION OF THE SYSTEM COMPONENTS}

\section{A. Mechanical to electrical energy conversion}

There are multiple possibilities for the conversion of the kinetic energy of the lotic water to electrical energy [1]-[3], [5]-[8]. One possibility is the use of small turbines, which are known from large hydroelectric power plants. On a small scale, which is the case for the proposed system, problems occur which are not known from large-scale applications, for example, the contamination or blocking of the turbine by carried debris. Because of this, another possibility of using the kinetic energy of the fluid has to be found. One solution is the use of turbulences of the water.

These turbulences are ever-present in most lotic waters. Nevertheless, the usability of artificial turbulences were investigated in a CFD simulation (ANSYS FLUENT).

If a solid body is exposed to a streaming fluid, a Kármán vortex street forms behind the body. This vortex street induces more or less periodic pressure differences, which can be used for a periodical bending of a piezoelectric bimorph (Fig. 3). It

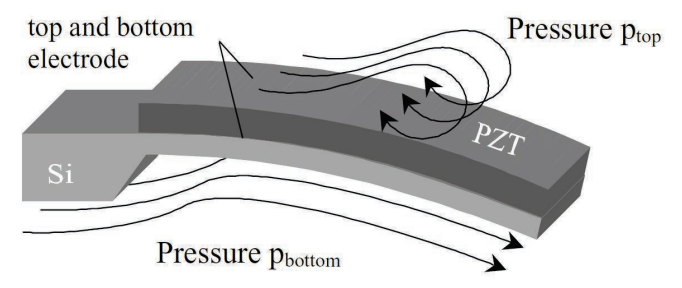

Fig. 3. Piezo bimorph generator [2]

was found in the CFD simulation (example shown in Fig. 4), that the pressure differences resulting from the Kárman vortex street are not sufficient to generate enough electrical power. These experiments were also carried out in the real world using a flow passage. Investigations from other research groups were either in another flow medium (air), or the approach was only discussed in theory. This work tries to fit this knowledge gap by simply using natural turbulences occuring in a real environment.

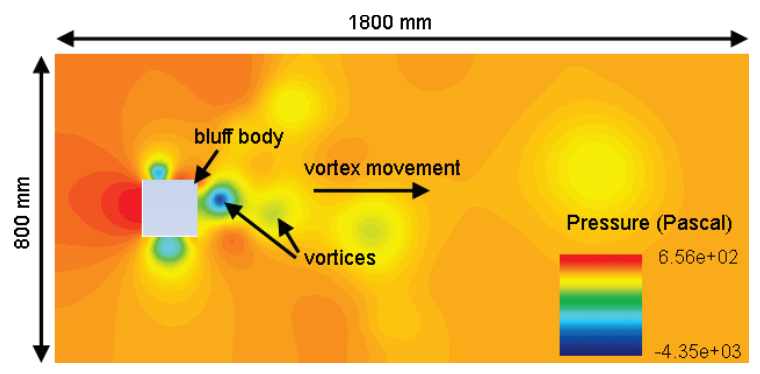

Fig. 4. Pressure at $t=2 \mathrm{~s}$, flow velocity $v=0.8 \mathrm{~m} \mathrm{~s}^{-1}$. The simulation was carried out without the attachment of the bimorph, as it was too complex to simulate the interaction of the fluid and the flexible structure at this point.

The advantage of the principles is that they are insensitive to contaminants or debris carried with the water. For the use in lotic water for a long time (years to decades) a redundant construction is needed, which can be realized by arranging multiple systems. The realized concept is shown in Fig. 5. One

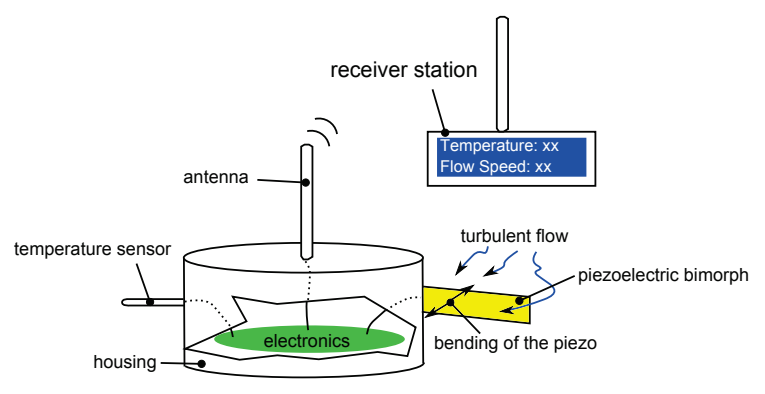

Fig. 5. Outline of the proposed system

step in the realization was a detachable but tight construction, especially at the clamp of the piezo. This was done by hardening silicone, which can be easily removed by peeling it of. For a later construction, resin can be used for better seal tightness.

\section{B. Processing and storage of harvested energy, microcon- troller and RF section}

The processing and storage of the harvested energy as well as the microcontroller and RF section is shown in fig. 6. The simplified principle of the storage of electric energy is shown in fig. 7. The piezoelectric bimorph delivers an $\mathrm{AC}$ voltage, which is being rectified and fed to the input capacitor. A low-power comparator compares the input voltage with a lowpower reference. If the voltage of the storage capacitor exceeds the reference voltage, current is delivered to the sink until a lower limit is reached and the cycle is started again.

If the data aquisition (in this case, the temperature measurement) and the transmission are inactive, the quiescent currents are as shown in fig. 8.

The energy consumption in active mode is dominated by the RF module (HOPE RF RFM02) [9]. A single data transmission (32 Byte at 19200 Baud) yields an energy consumption of 


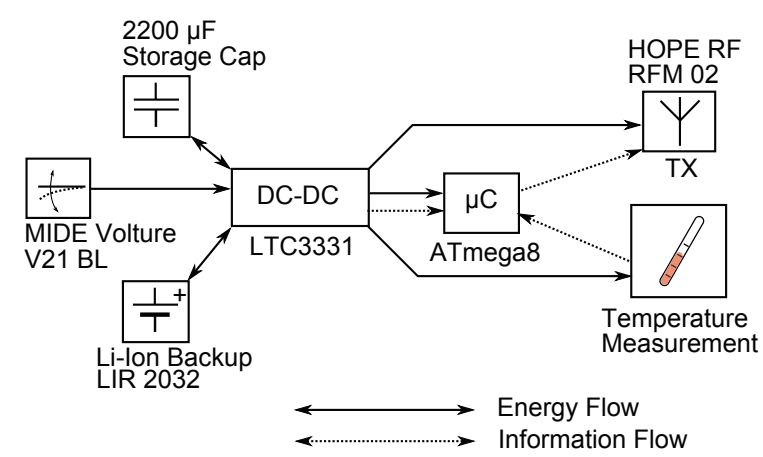

Fig. 6. Outline of the system's electronics

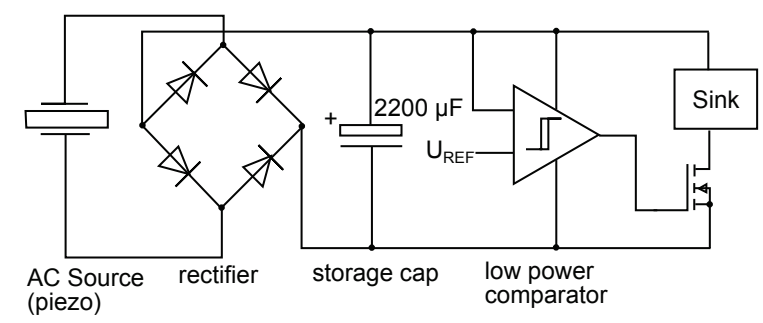

Fig. 7. Storage of piezoelectrically harvested power

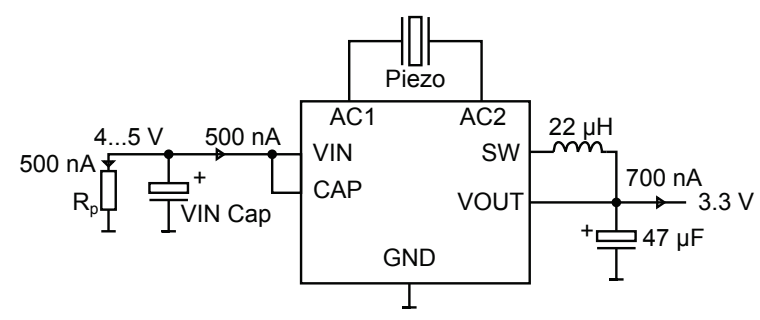

Fig. 8. Quiescent currents in the inactive mode $\left(\sum P_{i}=4.5 \mu \mathrm{W}\right)$

$866 \mu \mathrm{J}$ at an output power of $8 \mathrm{dBm}$. With this energy consumption, a continuous input power of $10 \mu \mathrm{W}$ and a quiescent power of $4.5 \mu \mathrm{W}$, every 157 seconds a data transmission is possible. The collection of input power can be extended to provide more power in larger intervals (for example data transmission via GSM).

\section{EXPERIMENTAL RESULTS}

We carried out experiments in the real environment at the river Würschnitz near Chemnitz, Germany. We measured in a rapid, the flow velocity was around $1.5 \mathrm{~m} \mathrm{~s}^{-1}$. The output power of the piezoelectric bimorph depends strongly on the turbulences in the water and its direction relative to the flow direction, see fig. 9. The output power was measured with a National Instrument measurement card. Fig. 10 shows the output power over time at an angle of $45^{\circ}$. More results are summarized in table I. These results show input powers over the limit of $4.5 \mu \mathrm{W}$. Nevertheless, at smaller flow velocities, a massive degradation in power was measured.

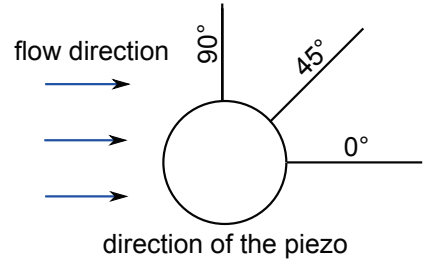

Fig. 9. Directions at which the piezo was tested

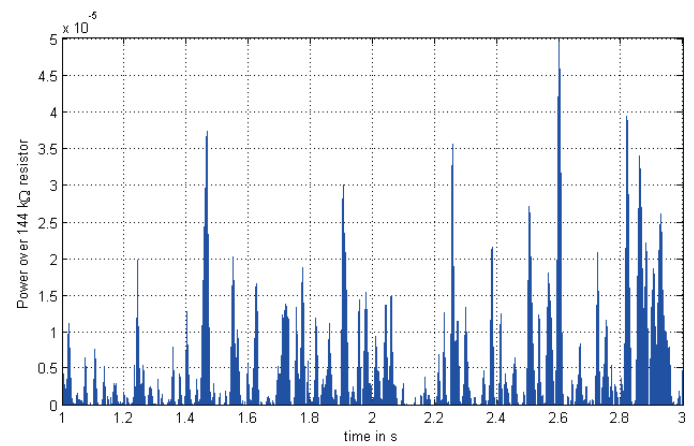

Fig. 10. Output power of the piezo when aligned $45^{\circ}$ to the flow direction

TABLE I

HARVESTED POWER UNDER DIFFERENT CONDITIONS

\begin{tabular}{|l|r|}
\hline Conditions & Mean power $(\mu \mathrm{W})$ \\
\hline \hline smooth, $0^{\circ}$ & 5.24 \\
\hline smooth, $45^{\circ}$ & 4.39 \\
\hline smooth, $90^{\circ}$ & 0.88 \\
\hline turbulent, $0^{\circ}$ & 2.96 \\
\hline turbulent, $45^{\circ}$ & 7.30 \\
\hline turbulent, $90^{\circ}$ & 6.09 \\
\hline
\end{tabular}

\section{NEXT STEPS}

Further developments can be divided in wireless communication and sensor system setup tasks. The sensor system setup can be miniaturised. Thereby a better enclosure especially for the electrical components is possible. Also the multiplication of the converter unit is thinkable to multiply the available energy to expand the application field. To be able to employ the energy harvester at small flow velocities (below $1.5 \mathrm{~m} \mathrm{~s}^{-1}$ ), improvements have to be done. For example, the commercially available piezo bimorph is very stiff and it has only a small area $\left(8 \mathrm{~cm}^{2}\right)$. This could be improved by attaching a larger flap. During the development process further promising methods were identified for coupling mechanical energy from the turbulent stream, which need to be investigated in detail.

For the wireless communication a real sensor network needs to be established to unlock the high potential of such systems.

\section{CONCLUSION}

In this work, a novel hydropower harvesting device and sensor node was proposed. During the development process a deeply examination of fluid mechanics within turbulent streams with different obstacles were carried out. Basing on 
FEM simulations and empirical tests using flow passages and real world tests a sensor system setup was designed, realized and successfully tested. The continuous power demand of approximately $4.5 \mu \mathrm{W}$ to run the electronics were generated down to a flow velocity of $0.5 \mathrm{~m} \mathrm{~s}^{-1}$. Besides the sensor housing mainly commercial components were used to build up the sensor system. During the empirical tests a couple of promising improvements were identified to increase the performances of the system significantly. The proposed sensor system enables an autonomously monitoring of lotic water quantities, e.g. temperature, within a cycle of approximately 15 minutes.

\section{ACKNOWLEDGEMENTS}

This contribution were elaborated within a scientific research seminar in the mechanical department of Technische Universität Chemnitz and we thank for the technical and the financial support. We thank the electrical department for the scientific support.

\section{REFERENCES}

[1] Taylor, G.W.; Burns, J.R.; Kammann, S.A.; Powers, W.B.; Welsh, T.R. "The Energy Harvesting Eel: a small subsurface ocean/river power generator", IEEE Journal of Oceanic Engineering, vol.26, no.4, pp.539,547, Oct 2001

[2] Pobering, S.; Schwesinger, N., "A Novel Hydropower Harvesting Device". Proceedings on International Conference on MEMS, NANO and Smart Systems, pp.480,485, 25-27 Aug. 2004

[3] Kwon, S.-D.: A T-shaped piezoelectric cantilever for fluid energy harvesting. Applied Physics Letters, 97, 164102 (2010)

[4] Kazmierski, T.J., Beeby, S.: Energy Harvesting Systems. Principles, Modeling and Applications, Springer New York, 2011

[5] Akaydın, H.D., Elvin, N., Andreopoulos, Y.: Wake of a cylinder: paradigm for energy harvesting with piezoelectric materials, Experiments in Fluids, 2010, Volume 49, Issue 1, pp 291-304

[6] Akaydın, H.D., Elvin, N., Andreopoulos, Y.: Energy Harvesting from Highly Unsteady Fluid Flows using Piezoelectric Materials, Journal of Intelligent Material Systems and Structures 2010 21: 1263

[7] Hobeck, J. D., Inman, J.: Artificial piezoelectric grass for energy harvesting from turbulence-induced vibration, Smart Mater. Struct. 2012 21:105024

[8] Li, S., Lipson, H.: Vertical-Stalk Flapping-Leaf Generator for Wind Energy Harvesting. In: Proceedings of the ASME 2009 Conference on Smart Materials, Adaptive Structures and Intelligent Systems, September 20-24, 2009.

[9] HOPE Microelectronics Co., Ltd.: Datasheet $433 \mathrm{MHz}$ Transmission Module RFM02.

[10] Jamil M. Renno, Mohammed F. Daqaq, Daniel J. Inman, On the optimal energy harvesting from a vibration source, Journal of Sound and Vibration, Volume 320, Issues 1-2, 6 February 2009, Pages 386-405

[11] A. Erturk, D.J. Inman: An experimentally validated bimorph cantilever model for piezoelectric energy harvesting from base excitations. Smart Mater. Struct. 2009 18:025009

[12] Kulkarni, R.V., Förster, A., Venayagamoorthy, G.K.: "“Computational Intelligence in Wireless Sensor Networks: A Survey", IEEE Communications Surveys \& Tutorials, vol.13, no.1, pp.68-96, doi: 10.1109 / SURV.2011.040310.00002, 2011

[13] Kumar, P.R.: "'Capacity, architecture, protocols, and sensing in wireless networks"', IEEE Information Theory Workshop, pp. 469- 472, doi: 10.1109/ITW.2004.1405349, 24-29 Oct. 2004

[14] Benecke, S., Reichl, H., Nissen, N.F.: "'Environmental comparison of energy scavenging technologies for self-sufficient micro system applications", IEEE International Symposium on Sustainable Systems and Technology, ISSST '09, pp.1, doi: 10.1109/ISSST.2009.5156712, 18-20 May 2009

[15] Kanoun, O.: "Energy-Management for Power Aware Portable Sensor Systems", Transactions on Systems, Signals and Devices, Vol. 3, No. 4, 2008, ISSN 1861-5252
[16] Kanoun, O., Keutel, T.: "'Energieautarke Sensorsysteme im Intelligenten Haus",, Expert-Verlag, S. 150-160, ISBN 978-3-8169-2789-1, 2007

[17] Chen, Z. S., Yang, Y. M., Deng, G. Q.: "'Analytical and Experimental Study on Vibration Energy Harvesting Behaviors of Piezoelectric Cantilevers with Different Geometries", IEEE, International Conference on Sustainable Power Generation a Supply, SUPERGEN '09, pp. 1-6, IEEE Press, New York, Dec. 2009 